\title{
Competent treatment Deficiency of Adhesion of Leukocytes at Children and Adults
}

\section{Dmitrieva Elena*}

Clinical Pharmacist, Charitable Pharmaceutical Clinic, Russia

*Corresponding Author: Dmitrieva Elena, Clinical Pharmacist, Charitable Pharmaceutical Clinic, Russia.

Received: May 08, 2019; Published: June 14, 2019

DOI: 10.31080/ASPS.2019.03.0312

"We cannot solve a problem, using the same mentality which we have created them"

A. Einstein

Opinion of the expert - of the Expert-Clinical of the Pharmacist.

Deficiency of adhesion of leukocytes - illness of long vaccination.

The foreword. At hit of microorganisms in a fabric inflammatory process begins. To one of its consequences is elimination leukocytes alien pftogen's and maintenance of a fabric homeostasis. That it has occurred, leukocytes need to carry out movings: at first on vessels, then it is direct in a fabric, and only then to finish with a microorganism. It is carried out by means of various auxiliary albuminous molecules - mediators inflammations, and also by means of various adgesines, necessary for the high-grade immune answer. Congenital absence of the last has been named by "deficiency of adhesion of leukocytes", and for today disease is considered incurable.

\section{Deficiency of adhesion of leukocytes (DAL)}

The rare hereditary disease extremely dangerous because of the hardest congenital immunodeficiency: fagocytar cages are incapable to get into fabrics, leaving cages defenceless before viruses and bacteria.

Now allocate three forms DAL: DAL-1, DAL-2 and DAL-3 (last also name "variant DAL-1").

To understand it, at first it is necessary to track the mechanism of transition of leukocytes in a fabric.

\section{Sticking of leukocytes to endotelium's}

For inflammation development it is necessary, that leukocytes not simply accumulated in a vessel gleam, but also entered close contact with endotelium and were ready to emigration for vessel limits in a fabric where there was an emergency.
In norm neutrophiles blood are concentrated in two compartments of a vascular channel. Their half freely circulates in an axial layer of a blood-groove, and half practically does not participate in circulation, forming wall a layer, adjoining to endotelium capillaries. For monocytes these indicators are even more displaced in advantage wall a layer.

At an inflammation the number of leukocytes in wall a layer can increase in tens times - the phenomenon of regional standing of leukocytes develops. It is connected with sharp delay of a bloodgroove in the expanded capillaries and venules and with growth of pasting ability of leukocytes. Glutinosity, or adhesiveness of an external membrane neutrophiles is in direct dependence on its activity. After stimulation neutrophiles start to be fixed strongly on endotelium's. Only after that they have an opportunity to creep further, in a fabric, the distress signal whence arrives.

Glutinosity of a membrane enfoteliocytes increases in a damage zone, that does contact endotelium's with leukocytes especially strong. It is found out, that adhesiveness endotelium's strongly varies under the influence of the products allocated neutrophiles which is going to enter with it contact. Among products - kationn fibers, leukotrienes, prostaglandin $\mathrm{E}$, biooxidizers $\left(\mathrm{H}_{2} \mathrm{O}_{2}, \mathrm{O}_{2}-\right.$ etc.). The factors released in units trombocytes (tromboxan A2, ADF, serotonine) also can give endotelium's the raised glutinosity.

At last, adhesiveness endotelium's depends and on its properties. At irritation endoteliocytes start to synthesise special glycoprotein - fibronectine. At the expense of it coupling between leukocytes and endotelium's becomes especially strong.

In a place of close contact of leukocytes with endotelium's there are favorable conditions for local increase of concentration mediators inflammations. In this connection permeability of microvessels increases in those places where there are the inflammation cages-effektors, ready to leave a vessel and to pass in a fabric. 
Emigration of leukocytes in the damage centre

After sticking of leukocytes to endotelium's capillaries their emigration for vessel limits soon begins. A stage of passage of a leukocyte through endotelium and bazal a microvessel membrane name - diapedesis - overshoot).

\section{Emigration depends on a kind of leukocytes}

- Polymorphonuclear leukocytes (PNL) pass through interendothelial cracks. In the beginning PNL passes to a junction a narrow shoot with which help adjacent cages - endoteliocytes are moved apart. Then cage contents are poured in pseudopod, taken root into a crack between endoteliocytes. Histamine and other factors of permeability reduce endoteliocytes and facilitate exit PBL from a vessel in a fabric (Chernuh A.M).

This traditional way of emigration not the unique

- PNL can get for vessel limits directly through a body endotelial cages. In a contact place endoteliocyte's with neutrophil's the membrane endotelium's is softened. Biooxidizers take part in this process $\left(\mathrm{H}_{2} \mathrm{O}_{2}, \mathrm{O}_{2}{ }^{-}\right.$and others), fosfolipazes, allocated neutrophil's in a zone close closing both types of cages. It conducts to that neutrophil as fails in cytoplasm endoteliocyte's. Thus endoteliocyte's does not suffer. Through bazal a membrane the leukocyte passes easily thanks to that in a place of contact to it the membrane passes from a gel condition in a condition colloid a solution. That is why preparations for treatment (the note of the author) are necessary, and it can be only herbs. After passage of a cage the membrane structure is restored (Cherly, Spector).

- Monocytes blood leave vessels the same by, as neutrophiles.

- Limfocyt passes through a body endoteliocyte's, being it is shipped in big vacuole. All these processes proceed, mainly, in capillaries and postcapillary venules.

In norm the considerable part neutrophiles and monocytes constantly leaves a blood-groove and moves in a fabric. In fabrics neutrophiles there live 5-7 days, and monocytes turn in macrophages and live weeks and even months without replacement with new cages. Instead of migrating from a bone brain in blood the same quantity of fresh leukocytes arrives. Having got to fabrics, leukocytes supervise a homeostasis.

\section{Chemotaxis}

At an inflammation in any site of a body speed of receipt of leukocytes in the damaged fabric considerably above, then in norm. The bone brain starts to throw out the reserves in blood. It conducts to leukocytoz's. At a sharp purulent inflammation the general number of leukocytes in blood usually increases for the account neutrophiles and their less mature forms (stick-core, young, mielocytes). In the centre of an inflammation term of a life of the leukocytes which have come from blood, too is reduced. If in the norm, the leukocytes which have left in a fabric move in different directions at an inflammation they actively move in strictly certain direction - towards the maximum concentration of substances drawing them - hemitrichapions (an armour. attractio - an attraction).

The directed migration of phagocytes has received the name Chemotaxis (taxis - an order). Without Chemotaxis there would be no inflammation as leukocytes - effectors would not gather in one place and would disperse on a fabric. Thanks to that migration of cages in the damage centre is focused in strictly certain direction, arises cellular infiltration and a series of the subsequent events connected with its formation. Active moving of phagocytes to a gradient direction - hemitrichapions occurs to the help of special ultrastructures of cages - microthreads. They consist from actinopodidae contractile the squirrel and settle down on periphery of cytoplasm of a phagocyte. At excitation of a cage of a microthread gather in units (bunches). At the same time other functions fagocytoz's after influence - hemitrichapions (ability to distinguish objects fagocytoz's to generate microbicid factors, secretion) do not suffer.

It is enough for excitation of the directed migration of leukocytes, that concentration - hemitrichapions at the face-to-face pole of a cage turned towards a vector of moving, was, at least, on $1 \%$ above, than at an opposite pole.

Gradient formation, hemitrichapions drawing leukocytes and the leader to development inflammatory infiltration's.

- Many components of plasma filtered in a fabric, possess chemotactic properties. Substances which are formed in the course of blood curling - kallicrein concern them the activator of plasminogen's, fibrinpeptid B, C3a - and C5a - fractions of complement. From antibodies G rift peptid-lejkoagressiny with expressed chemotactic properties. 
- Hemitrichapions are formed at destruction of cellular membranes. At oxidation arachidon acids appear leukotriens B4, C4 and D 4, and other derivatives sharply strengthening chemotaxis of leukocytes in the centre.

- Having appeared in the damage centre, leukocytes allocate substances which support a high gradient - hemitrichapions. So lisosomal proteazes neutrophiles to straight lines by activate complement, and during this activation are formed - hemitrichapions - Сза, C5a, etc. Neutral proteazes as collagenaza and elastaza destroy fibrous structures of a connecting fabric - collagenic and elastin threads. Products partial destruction collagen and elastin serve hemitrichapions not only for PNL, but also for monocytes.

Attraction neutrophiles cause also products of other cages from the inflammation centre. So, monocytes are secreted the lowmolecular factor with weight 400-600 dalton's. On the properties it differs from C5a.

Limfocytes after their stimulation by a specific antigene or nonspecific mitogenes (lectines - phytohemagglutinin (FA), concanavalin (Con, etc.) are secreted the fibers drawing PNL in the centre.

It is important to underline, that - hemitrichapions not simply involve -PNL in a damage zone, but also activate them. The last is connected by that on membranes neutrophiles there are special receptors to - hemitrichapions. Is better from them receptors to microbic - hemitrichapions are studied. From them the active fraction - formylmethionilpeptid which causes powerful chemotaxis PNL and monocytes is allocated.

In the centre of an inflammation phagocytes in reply to stimulation start to throw out in environment the lizosomal granules. This process has received the secretion name. In lizosomas there is a whole set mediators inflammations.

Degranulation it is connected with activity of microtubules. On them lizosomas move from the cage centre, from near-nuclear zones, - to an external membrane. After merge to a granule membrane are emptied on Wednesday. At other variant they can be pushed out from a cage entirely. Mediators inflammations can be allocated from phagocytes not only in a course secretor degranulation, but also at destruction of phagocytes.
Having appeared in the centre, neutrophiles work not alone, and in cooperation with other types of cages. The important role is played by interactions neutrophiles with limfocytes, neutrophiles with monotsitami-macrophages, neutrophiles with corpulent cages. Limfocytes in the inflammation centre allocate soluble factors which strengthen microbicid functions neutrophiles, do their more capable in struggle against (A.N.Majansky's) microbes. Along with it B-limfocytes and plasmatic cages allocate specific antibodies. Formed complexes irritate an antigene-antibody neutrophiles and strengthen them effector functions in the inflammation centre. It is often observed at an allergic inflammation.

In the latter case in the centre can migrate much eozinofiles. At contact of cages-targets, sensibilization with Ig E-antibodies with specific allergen it is allocated special eozinofil-hemotaxiche the factor, nearby 500 dalton's (ECF-A). Their basic function consists that they will neutralise histamine and others mediators the inflammations allocated with various types of cages-effektors.

\section{Fagocytoz}

After an exit from a vessel in the centre of damage leukocytes begin to englobe microbes. The phenomenon fagocytoz's has been in detail described still I.I.Mechnikovym in the end of the last century.

\section{Fagocytoz includes some stages}

1. Recognition (cognizance) a phagocyte of the object which is subject to absorption;

2. An object attachment fagocytoz's to an external membrane of a cage and its immersing as a part of gemmated vacuole (phagosomes) in cytoplasm;

3. Microbe killing in phagosome's and its subsequent digestion;

4. Allocation of the rests from the digested microbe in the extracellular environment.

For linkage of microbes on a membrane of phagocytes there are special receptors to a Fc-fragment of an antibody and to the Cz-component complement's. In reply to hit of microbes in an organism are formed antimicrobial antibodies Ig M and an Ig Gclass. They sorbed on a microbe surface. In a case sorption Ig Mantibodies to them the Sz-fraction complement's in addition joins. The phagocyte connects in a complex - a microb+Ig G-antibody or 
microb+lg M-antitelo+complement through the receptors specified above. Thus, antibodies act in a role of opsonins - the factors facilitating fagocytoz.

The same mechanisms work not only at fagocytoz's microbes, but also other particles (old eritrocytes). Properties of opsonins the products formed from Ig G under action proteaz possess also. Along with it opsonines can and not to be connected with antibodies and complement's. In plasma special a2-globulin which is easily besieged from a solution on a cold contains, therefore it named cryoprecipitation globulin. This fiber sharply raises ability of phagocytes to absorb microbes and other particles, alien for an organism.

After the object will be fixed on a phagocyte membrane, formation fagocytar vacuole's, or phagosomes begins. In formation phagosomes participate contractile fibers of a phagocyte. On properties they remind actin and myosin from muscular cages. Unlike a muscle in a phagocyte actin in itself does not activate ATF-pase, connected with myosin's, and can so to arrive only in the presence of other fiber - cofactor's. Along with it in phagocyte cytoplasm there is the special fiber connecting threads actin's in bunches - actinic binding fiber. Actin turns to gel then business enters myosin with cofactor's. Myosin and cofactor in the presence of $\mathrm{Mg}^{2}+$ and ATF reduce gel actin's, transforming it in compact units.

If the gel which is exposed to reduction, is attached to a plasmatic membrane of a phagocyte from within against object phagocytosis deepening is formed, and the object is surrounded with cytoplasm ledges - pseudopodiums and grasped in it. In phagosoma's, containing a microbe, a series of events which conduct to destruction of the activator and its the subsequent destruction is played. Microbicid effects in such cases are connected with activity NaDF-NaDFH-oksidaz and others fermentative systems which participate in formation $\mathrm{H}_{2} \mathrm{O}_{2}$, superoxidanion's $\mathrm{C}_{2}$ ), singlet oxygen $\left(10_{2}\right)$ and other biooxidizers. These fermentative systems are included in a membrane phagosomes. At their weak functioning the microbe has an opportunity free to live in a phagocyte, and the sharp inflammation passes in the long form. That is why antioxidants are necessary, but I believe, in a phase of rehabilitation of illness. (The note of the author).

In a phagocyte vacuole with the killed microbe should merge (conjugate) with lysosoma's. Only object digestion phagocytosis in this case will begin. In most phagosome's hydrolytic enzymes does not contain. After connection phagosome's with lysosoma's it is formed difficult vacuole - phagolysosome in which the killed microbes are exposed to attack from the party lysosomal a hydromanhole. In phagolysosomes it is fuller developed microbicid cage potential, therefore in some cases microbes keep ability to live in phagosomes, but quickly perish after merge phagosomes with lysosoma's. At immunity decrease at long vaccination these processes are broken up to full not abilities to struggle with any infection (the note of the author).

The third component of inflammatory reaction is proliferation at an inflammation. It begins already from the very beginning of an inflammation.

Proliferation (prollferatio, occurs from an armour. Words proles - posterity and ferre - to create) at an inflammation represents local reproduction of cages in which various fabric components take part.

Reparative processes in fabrics proliferation cages at an inflammation have strict sequence and various fabric components participate in it.

Proliferative processes proceed especially actively after tearing away necrotic weights and destruction of pathogenic agents. In the conditions of an inflammation the damaged fabrics and, especially, blood cells are sources gumoral the factors stimulating damage of fabrics. Proliferation at a sharp inflammation at the closing stages of its development it is similar to healing of a wound after fabric damage.

If defect includes an integumentary fabric, for example, derma's dynamics of process has following features.

In the inflammation centre there is a fibrin quantity. From wound edges epidermis grows deep into, densely adjoining to healthy derma's from different directions thanks to fibrin. In 1-2 weeks, depending on the sizes of defect, epidermis in the heart of a wound forms continuous epitelial a layer.

Especially with this process the connecting fabric thanking proliferation fibroblasts and to fabric development is restored also. As the basic source fibrinoblasts and capillaries the hypodermic fabric which is richly supplied by capillaries serves and has the big number pericytes, - low-grade cages. In the conditions of an 
inflammation when the considerable quantity of growth factors is formed, occurs formations fibroblasts and capillaries, possibly, from pericytes. This process most actively passes in depth of a wound where the greatest quantity of connecting structures which in process of growth carry out a crack bottom, paved epitelium's is formed, lifting it to surface level. Epidermis during long time remains thin. Growth epitelium's and reproduction of connecting structures is regulated by numerous general and local factors.

To local factors concerns - size of a blood-groove and growth factors, the general - hormones, mediators and other intermediaries neuroendocrine regulation of inflammatory process. On an outcome reparative processes, the hem condition, the big influence renders a condition immunobiological mechanisms, therefore it is necessary to do in dynamics Immunogramms (the note of the author).

The present stages - the Mechanism of penetration of leukocytes in the inflammation centre

Leukocytes are activated in an alteration phase various chemoattractant (from group mediators inflammations) which migrations of leukocytes and to their subsequent exit in a fabric further promote.

\section{Two groups attractant allocate}

- "Classical" attractants - N-formilnye bacterial peptid antigenes, fibers of system complement's C3a and C5a, and also various lipid molecules on type leukotrien's B4.

- "Selective" attractants - group of fibers of superfamily chemokines which can be divided into 4 groups conditionally: CXC, CX3C, CC and C (are named so on presence of the two first rests cisteine's - steam rooms and highly conserved).

Chemoattractant communicate with specific receptors of leukocytes and cause their moving on «to a gradient chemoattraction», - to a place of the centre of an inflammation. At the person it is known nearby 18 chemocins receptors, all of them are connected with G-fibers and consist from 7 transmembrane domains that is typical for many receptors. At activation of these receptors the group of molecules of adhesion which is necessary for consecutive cascade transition of leukocytes in a fabric starts to be synthesised.
Initially leukocytes move with a blood current, gradually leaving an axial stream on periphery and coming nearer to a vessel wall (marginalization) then leukocytes are attached to endotelium's and filter through it directly to a damage place.

Classically allocate three stages in the course of achievement of the fabric centre of an inflammation

Swing caused selektins; activation of leukocytes under influence chemokins and introduction in a fabric, mediated integrins.

The first stage remains swing or rolling the leukocytes, caused by special fibers - selektins and integrins.

Selektins it is possible to name recognition and attachment molecules. The majority of leukocytes are secreted selektin-P, cages endotelium's (inflamed) - selektins E and L. The basic receptor for all of them - P-selektins glycolisate a receptor-1 (PSGR-1). At the expense of it leukocytes are capable to be attached strongly to endotelium's; thanks to it leukocytes which do not possess necessary «receptor the passport», cannot pass in the inflammation centre.

Integrins - one more group of the fibers possessing adhesive properties.

In their group allocate two cores subfamilies

$\beta 1$ - and $\beta 2$-integrins, also there are also others.

The most important are following representatives

- $\quad \alpha$ 4ß7-integrin - it is necessary for an attachment to receptors of cellular adhesion mucous vessel MADCAM-1 (English mucosal vascular addressin cell-adhesion molecule-1);

- $\quad \alpha 4 \beta 1$-integin or VLA4 (English very late antigen-4) - it is necessary for an attachment to an extracellular receptor of adhesion VCAM-1 (English vascular cell-adhesion molecule-1);

- $\quad$ 2-integrin - it is necessary for connection with an endocellular receptor of adhesion ICAM-1 (English intracellular adhesion molecule-1).

- $\quad \alpha$ L $\beta 2$-integrin, having abbreviation LFA-1 (lymphocyte function-associated antigen-1) which also contacts ICAM-1 a receptor.

All these complexities are necessary to a leukocyte to pass in a phase slow swing, and then and at all to stop. 
Capture of leukocytes occurs by strong linkage integrins to fibers VCAM and ICAM (them carry to superfamily of antibodies, are synthesised endoteliocytes under influence mediators inflammations).

That there was a strong linkage (adhesion), moleculesregulators - chemokines (the cores - IL-4, FNT) also are necessary. They can contain in a blood-groove, be transported endoteliocytes or be synthesised activated trombocytes and corpulent cages. Also these chemokines possess high affinity to the mentioned receptors, associated with G-fiber (GPCR - G-protein coupled receptor).

Conditionally it is considered, that $\beta 1$-integrins - molecules eosinofiles, monocytes and limfocytes, contact VCAM, and $\beta 2$ - integrins - fibers neutrophiles and basofiles - with ICAM; with their help the leukocyte is strongly attached to endotelium's. After that the final stage - transmigration begins.

Penetration through a vessel wall - extremely difficult process. It begins with slow moving or phagocyte crawling on endotelium's (with the help macrofagal an antigene 1 - MAC1). The leukocyte «searches» for a convenient place for migration in a fabric. To migrate a leukocyte it is capable as through intercellular space (in most cases), and it is direct through a cage (such is the mechanism of transmigration of leukocytes in CNS).

Between endoteliocytes the leukocyte gets thanks to the extremely flexible membrane, it «filters» through intercellular connections literally. This process is long enough and combined, occupies 15-20 minutes and is regulated by set chemokines, cytokines, etc.

Transcellular (it is direct through endoteliocyte) the phagocyte passes by means of recently open transport mechanism - vezikulvakuoljar organelles (VVO). These are small membrane structures, presumably specialised conveyors of leukocytes (neutrophyles) through a body endoteliocytes.

Multistage process of migration of a leukocyte through a vessel wall on an example neutrophyle.

At first the leukocyte is rolled on a surface endotelium's, then becomes activated and adheres to endotelium's, then passes through it, gets through bazal a membrane and migrates in a site of damage of a fabric under action - hemitrichapions. At different stages of this process different basic molecules work: selektins - at rolling's, chemokines (usually presented to the connected kind with proteoglicanes) - at activation neutrophyles for affinity increase integrins, integrins - for proof adhesion, CD31 (PECAM-1) - for transmigration. Neutrophyles express low levels L-selektin's; they contact endotelium's mainly through R - and E-selektin's. ICAM-1 - a molecule of intercellular adhesion 1; IL - interleukin; PECAM-1 - trombocytar an adhesion molecule endothelial cages 1; TNF - the factor necroz's tumours.

Having overcome endotelium, fagocytar the cage appears in the inflammation centre where puts things in order and promotes fabric restoration.

From described above the mechanism of activation of leukocytes and their penetration into the inflammation centre importance of correct work of all involved molecules becomes clear.

But owing to a certain genetic mutation adhesive ability of leukocytes can be broken - such condition is called as deficiency of adhesion of leukocytes (DAL).

Classify DAL exclusively by the form defective molecules (and genes, their coding). The clinical picture, the approach to diagnostics and possible treatment (or to disease knocking over) are identical in general though there are also nuances.

\section{Deficiency of adhesion of leukocytes of 1 type}

DAL 1 type - the rare disease transferred on autosom-dominat to type of inheritance. This primary immunodeficiency is coded by gene ITGB2 which is responsible for an expression $\beta 2$-integrins (CD11/CD18). Prevalence of disease - 1:1 000 000, in the literature it is possible to meet all about 300 cases.

For the first time about it have informed as on high-grade independent disease in 1997 though in the literature and earlier cases of immunodeficiencies because of defect $\beta 2$-integrins have been described. Most of all suffer neutrophiles which are not capable to get into a fabric.

Clinically it is shown recurrent life-threatening by bacterial and mushroom infections. Frequently the immunodeficiency proves very much early, and infections quickly pass in the septic form.

Distinctive feature of any DAL - absence of pus in the inflammation centre. On a skin and mucous the centres necroz's are found out, at children's age death rate is highest. Further, (if competently 
to eliminate all infections), illness proves not so sharply, more adult patients suffer from heavy gingivitis and a chronic periodontitis, but probability of a lethal outcome at them it is essential lower.

Diagnostics can be carried out by methods of gene engineering, but for DAL-1 it is inexpedient. Reveal markers CD11/CD18 a method flowing cytometria's - habitual and even the routine analysis in immunology is much more often. Accordingly, in the absence of the given markers it is possible to speak about DAL 1 type. Diagnostics of markers (the note of the author) is necessary.

Unique method of treatment DAL-1 at present is allogenic transplantation of haemopoetic cages (ATHC). On an extent with 1993 for 2007 ATHC it has been made to 36 patients at the age from 2 months till 14 years (middle age - 9 months). The survival rate has made about $75 \%$. It is important to notice, that the most part of death was the result of impossibility to find the HLA-compatible donor.

Besides it, there is perspective enough pathogenetic therapy, namely - a transfusion granulocytes. A method for today disputable enough, certainly, to eliminate it DAL is not capable, but can quite cope with heavy infections, as, for example, with gangrenous eczema.

At last, DAL-1 it is possible to stop and constrain by means of competent Antibiotic therapy, and it is that case when antibiotics should be applied and as preventive maintenance.

Deficiency of adhesion of leukocytes 2 types

At the heart of DAL-2 - defect selektines and, as consequence, impossibility «rolling» leukocytes on endotelium's. But, unlike DAL-1, the immunodeficiency aetiology not so is obvious, it is known only about 10 cases of the given disease.

Molecular nature DAL-2 - insufficiency of fiber-carrier GdF-fukoses (fucosylation - one of necessary updatings transmembrane adhesion receptors); fiber a carrier - GFTP (English GDP-fucose transport protein) is coded by gene SLC35C1, it FUCT1.

Also, deficiency aforementioned GFTP leads to two more rare phenomena: infringement of synthesis of antigenes of ABO-system (the Bombay phenomenon) and insufficiency of antigenes of system of Lewis. Thus, defect of one gene FUCT1 leads at once to three rare pathologies which extremely hard give in to updating.
Clinical picture, strangely enough, frequently softer in comparison with DAL-1. Leukocytes though are deprived correctly working selektines, nevertheless have absolutely intact integrines, that is, at least any part of phagocytes nevertheless is capable gets into a fabric and renders protection against an infection which become less serious, than in the previous case. The immunodeficiency is shown basically on the first year of a life, in more adult period of complication not so are expressed (though there is a chronic periodontitis).

Insufficiency all the same conveyor GFTP can be shown (though and is rare) a growth inhibition and intellectual backwardness.

To diagnose DAL-2 it is possible on the basis combination a phenomenon of the Bombay group of blood and absence of antigenes of Lewis; the genetic analysis of gene FUCT1 will be the definitive proof of existence of a pathology.

Treatment DAL-2 usually proceeds without results. For today besides symptomatic treatment by antibiotics the medicine has nothing to offer, in general. The only thing reception L-fukoses oral can be more or less effective. At least, in research the condition of patients has quickly improved; however after a month neutrophiles have ceased to react to similar support.

\section{Deficiency of adhesion of leukocytes 3 types}

Often DAL-3 carry to versions DAL-1 of type, however, such approach hardly the correct. DAL-3 much more aggressive disease which it is practically impossible to stop.

The illness aetiology consists in defect of gene FERMT3 which is responsible for synthesis of all $\beta$-integrins. Full inability of a leukocyte to though to any attachment to endoteliocytes, let alone penetration through a vessel wall becomes result.

Besides usual heavy infections patients suffer also hemorragh a syndrome (thrombocytopenia of Glazman - duration of a bleeding makes more than 15 minutes at norm of 2-6 minutes It it is quite expected as trombocytes in the course of aggregation use $\beta 3$ integriny.

DAL-3 for today it is revealed approximately at 20 families all over the world. It is the heaviest form DAL with high letal, frequently newborns in such families very quickly died, and even before diagnosis statement. 
In addition to already described clinical picture (recurrent heavy infections, absence of pus, hemorragh a syndrome) it is possible to name defect of a bone fabric like osteopetrosis (an osteosclerosis, marble illness). Comes to light not at all patients, however, the similar pathology can essentially help with diagnosis statement.

Actually, diagnostics is spent by means of the gene analysis. There is a number of tests in which it is possible to distinguish defect neutrophiles, for example, the test with neoconservatism simhasan's which will help to differentiate DAL-3 from DAL-1. However similar tests demand time, and disease frequently does not leave chance of meditations (the note of the author).

Treatment differs from that at other forms DAL a little. These are transfusions granulocytes and (in this case) trombocytes, and the number of transfusions can reach 50 in a year. In the unique way aetiotropic therapy still has allogenic transplantation of haemopoetic cages [1-23].

\section{Conclusions}

1. As the first vaccine which enter into an organism of the child - BCG it does not finished phagocytoz then the organism of the child cannot resist to an infection, arises illness (details in articles about treatment of a tuberculosis at children). The author has found one preparation, which was used for tuberculosis treatment (except tuberculoma's) at children of 7-10 years with positive results. Against reception tableted Sangviritrine's the tuberculosis recovers. It is simultaneously possible to block action of vaccine BCG.

2. Except Sangviritrine's candles and pricks Immunofan's were appointed. Preparations it is transferred well by children.

3. At adults - pricks Derinat's not only stabilise a condition, but also do normal Immunogramm's.

4. The note: At this disease it is preparations of a choice for treatment. They are well transferred, but always it is necessary to do Immunogramms 3 levels of complexity with compliment definition, except children till 3 years. To 3 summer age of an inoculation it is impossible to do. It is impossible to do them and after 50 years at adults.

5. At vaccination also there is an occurrence of syndromes - strengthenings of an infection and imprinting's, a part children die in a dream - a syndrome of sudden death at children in a dream from vaccines as a result of a respiratory distress-syndrome, therefore I advise not to do them till 3 years precisely, and further - too it is better not to do! At least, it is necessary to look on a situation of health of the child. Also it is inadmissible vaccines to do violently or a deceit as it do in Russia.

6. After a course curing, Antioxidants as there is a failure and in it to system of an organism of children are necessary necessarily. These are oxygen cocktails, herbal medicine colloid solutions of extracts from herbs).

7. By me it is developed aetiotropic specific treatment of some children's infections without vaccines. These are such, as a tuberculosis, a measles, rubella. I can give the book and on other diseases. After all after disease proof immunity after illness develops. From vaccines it is possible to be ill many times.

8. I can publish the book on competent treatment in Immunology. It is important for children and adults. Competently picked up Immunomodulators, - can cure conservatively any illness. But they have also by-effects. Too it is necessary to remember it!

\section{Bibliography}

1. E Schiffmann., et al. "N-formylmethionyl peptides as chemoattractants for leucocytes". Proceedings of the National Academy of Sciences of the United States of America 72.3 (1975): 10591062.

2. C Murdoch and A. Finn. "Chemokine receptors and their role in inflammation and infectious diseases". Blood 95.10 (2000): 3032-3043.

3. M Baggiolini., et al. "Human Chemokines: An Update". Annual Review of Immunology 15.1 (1997): 675-705.

4. EC Butcher. "Leukocyte-endothelial cell recognition: Three (or more) steps to specificity and diversity". Cell 67.6 (1991): 1033-1036.

5. K Ley., et al. "Getting to the site of inflammation: The leukocyte adhesion cascade updated". Nature Reviews Immunology 7.9 (2007): 678-689.

6. J J Campbell., et al. "Chemokines and the arrest of lymphocytes rolling under flow conditions". Science 279.5349 (1998): 381384. 
7. A M Dvorak and D Feng. "The vesiculo-vacuolar organelle (VVO): A new endothelial cell permeability organelle". Journal of Histochemistry and Cytochemistry 49.4 (2001): 419-431.

8. Hamid Nawaz Tipu. "Leukocyte Adhesion Deficiency Type I: A Rare Primary Immunodeficiency Disorder". Pediatric Allergy and Immunology 28.3 (2017): 303-305.

9. E. Almarza Novoa., et al. "Leukocyte adhesion deficiency-I: A comprehensive review of all published cases". The Journal of Allergy and Clinical Immunology 6.4 (2018) 1418-1420.

10. T W Kuijpers., et al. "Leukocyte Adhesion Deficiency Type 1/ Variant: Dysfunctional b 2 Integrins". Journal of Clinical Investigation 100 (1997): 1725-1733.

11. E van de Vijver., et al. "Leukocyte Adhesion Deficiencies". Hematology/Oncology Clinics of North America 27.1 (2013): 101116.

12. M Movahedi., et al. "Clinical and laboratory findings in Iranian patients with leukocyte adhesion deficiency (study of 15 cases)". Journal of Clinical Immunology 27.3 (2007): 302-307.

13. W Qasim., et al. "Allogeneic Hematopoietic Stem-Cell Transplantation for Leukocyte Adhesion Deficiency". Pediatrics 123.3 (2009): 836-840.

14. F Mellouli., et al. "Successful treatment of fusarium solani ecthyma gangrenosum in a patient affected by leukocyte adhesion deficiency type 1 with granulocytes transfusions". BMC Dermatol 10 (2010): 2-4.

15. B Ma., et al. "Fucosylation in prokaryotes and eukaryotes". Glycobiology 16.12 (2006).

16. K Lühn., et al. "The gene defective in leukocyte adhesion deficiency II encodes a putative GDP-fucose transporter". Nature Genetics 28.1 (2001): 69-72.
17. A Hidalgo., et al. "Cunningham-Rundles, and P. S. Frenette, "Insights into leukocyte adhesion deficiency type 2 from a novel mutation in the GDP-fucose transporter gen". Blood 101.5 (2003): 1705-1712.

18. T Marquardt., et al. "Leukocyte adhesion deficiency II syndrome, a generalized defect in fucose metabolism". Journal of Pediatrics 134.6 (1999): 681-688.

19. S Yakubenia and M K Wild "Leukocyte adhesion deficiency II: Advances and open questions”. FEBS J 273.19 (2006): 43904398.

20. K Jurk., et al. "Novel integrin-dependent platelet malfunction in siblings with leukocyte adhesion deficiency-III (LAD-III) caused by a point mutation in FERMT3". Thrombosis and Haemostasis 103.5 (2010): 1053-1064.

21. S Schmidt., et al. "Kindlin-3-mediated signaling from multiple integrin classes is required for osteoclast-mediated bone resorption". Journal of Cell Biology 192.5 (2011): 883-897.

22. E F Goljan. "Pathology". Chapter 3 (2012).

23. Kuebi (Kübelbeck) B: Nature Reviews immunology 7 (2007): 679.

\section{Volume 3 Issue 7 July 2019}

(C) All rights are reserved by Dmitrieva Elena. 